\title{
Estimation of EOP from VLBI: Direct Approach
}

\author{
L. Petrov \\ Code 926, NVI, Inc./NASA Goddard Space Flight Center, Greenbelt, \\ $M D 20771$ USA
}

\begin{abstract}
The currently adopted strategy of EOP estimation from VLBI is to estimate six parameters: UT1, UT1 rate, pole positions and nutation offsets for each 24-hour session independently. Then the resulting time series of raw Earth orientation parameters are filtered and a regression analysis is performed in order to obtain nutation coefficients, polhode of the pole and other physical parameters. Thus, the latter parameters are obtained indirectly in two stages. An alternative approach of direct estimation of the final Earth orientation parameters is presented. Pole coordinates and UT1 are considered as a sum of three components: the low-period component which is modeled by a cubic spline, the harmonic component which includes forced nutation, precession and subdaily variations of EOP, and the stochastic component which is modeled by a linear spline with segment length 1-2 hours. All parameters are obtained in a single LSQ solution using all available data.
\end{abstract}

\section{Introduction}

Rotation of the Earth is a complicated process. We don't observe it directly, but estimate parameters of the model of indirect observations. The choice of the set of parameters is inspired by both theory and our ability to estimate parameters of the model. Earth rotation in the reference system tied to the rotating Earth can be represented by a superposition of three components: 1) low-frequency variations (period $>2^{d}$ ), 2) quasi-diurnal and quasi-semidiurnal variations, and 3) a stochastic component.

The traditional approach of EOP estimation from VLBI is first to estimate six parameters: pole coordinates, UT1 and its rate, nutation offset angles for each 24-hour session independently, then to filter the raw time series of the estimates, and then to perform statistical analysis of the resulting time series. High-frequency Earth orientation variations cannot be determined in this way, and they are obtained in a different solution.

The traditional approach has an internal contradiction. If we assume that nutation is a purely harmonic process, with known frequencies, which corresponds to a quasi-diurnal polar motion then we need to estimate only amplitudes and phases of the harmonic components, but not to represent nutation angles as a time series. If we assume that there are non-harmonic components in nutation, e.g. free core nutation, with a broad spectrum, then the traditional 
procedure cannot strictly separate them from pole coordinates. The deficiencies of the traditional approach are:

- The components of the retrograde quasi-diurnal polar motion (nutation) with frequencies rather far away from the diurnal frequency are not perfectly separated from the long-period components of polar motion. As a result the estimates of their amplitudes are corrupted.

- The correlations between the estimates of daily nutation offsets are neglected, and series are usually treated as statistically independent.

- Nutation, precession, high-frequency EOP variations are treated differently.

\section{Direct approach}

An alternative way is to estimate all components of EOP in a single global solution. Since the length of the data set of regular VLBI observations started in January 1984 is increasing, it is already feasible to separate aliases related to the 18.6-year period. Not only the main components of nutation but almost all terms can be determined from the observations. The following parameterization is proposed:

- $X_{p}, Y_{p}, U T 1$ and their rates for each session. They model slowly varying components.

- Set of sin- and cos- terms for $X_{p}, Y_{p}$ and $U T 1$ at frequencies within the diurnal and semidiurnal bands (with respect to the rotating Earth). They include precession, CEP offsets, nutations, tidal diurnal and semidiurnal EOP variations, harmonic variations at ad hoc frequencies. The frequencies are taken from theory.

- Parameters of a linear spline for $X_{p}, Y_{p}$ and $U T 1$ with segment length 1.5 hours. They model the stochastic component in the Earth's rotation.

- Other parameters.

Such a choice of parameterization should adequately model all constituents of Earth rotation. However, these parameters create some difficulties:

- The theoretical spectrum of EOP variations has closely-separated components (multiplets) which cannot be resolved using the given data set.

- There are synonymous constituents, e.g. nutation and diurnal high-frequency variations in polar motion.

- Three constituents are overlapping and are not independent:

- there is a linear dependence between a session-average parameter and an average value of linear spline; 
- there is a linear dependence between a session-rate parameter and a rate of change of the linear spline;

- there is a quasi-linear dependence between a harmonic parameter and coefficients of the linear spline.

Fortunately, we can overcome these problems. The lowest critical frequency is assumed. The harmonic variations with the frequency lower than the critical frequency are considered as a secular change and they are represented by a linear model. The harmonic constituents with frequencies which differ by less than the critical frequency are grouped into multiplets. It is assumed that the frequencies of the components of a multiplet and the ratios of the amplitudes are known from theory. The complex admittance of the multiplet is estimated instead of the amplitude and the phase of a singlet. Strong de-correlation constraints on the coefficients of the linear spline for each session are imposed: a) the average is zero (de-shifting), b) the average rate is zero (de-trending), c) the amplitudes of sinand cos- components at the specified frequencies are zero (de-harmonization).

\section{Solution}

The approach outlined above has been implemented in the CALC/SOLVE program package. I used all available VLBI observations for 1979.7-2000.0, 2.8 $10^{6}$ observations, 2961 sessions.

The estimated Earth orientation parameters were:

- 736 harmonic parameters including precession rate and obliquity rate, celestial pole offsets, 148 nutation frequencies, 34 additional tidal frequencies, 3 ad hoc frequencies. The lowest frequency was $1 \cdot 10^{-8} \mathrm{rad} / \mathrm{sec}$ (period of 18.6 years)

- 2559 daily pole coordinates and UT1 as well as their rates.

- 44150 parameters of linear splines for pole coordinates and UT1. The duration of a span between nodes of the spline was 90 minutes.

- Other parameters: station positions and velocities, source coordinates, troposphere path delay, troposphere gradients, clock parameters.

Weighted root mean square of the postfit residuals is 24.6 psec. Formal uncertainties of the estimates of harmonic variations are $20-300$ prad. The largest correlation of 0.91 is between the 18.6-year nutation term and precession. Only 89 correlations out of 270480 are greater than 0.7 .

Comparison with the empirical nutation coefficients obtained by Souchay et al. (1995) and with semi-empirical coefficients IERS96 (1996) showed the differences are small. They don't exceed 1-2 nrad, but they are not negligible. Some terms are presented in Table 1. Plots of the estimates of the stochastic component in Earth rotation are presented in Figure 3.. 
Table 1. Differences in some terms with respect to the IAU 1976 precession, IAU 1980 nutation models.

\begin{tabular}{|c|c|c|c|c|}
\hline Term & D irect solution & Souchay (1995) & IERS96 & Units \\
\hline Precession rate & $-2.95 \pm 0.02$ & -3.00 & -2.96 & $\mathrm{mas} / \mathrm{yr}$ \\
\hline Obliquity rate & $-0.27 \pm 0.009$ & $-0.26 \pm 0.01$ & -0.23 & $\mathrm{mas} / \mathrm{yr}$ \\
\hline$\Delta \Psi$ (in) $\operatorname{Per} 18 .^{a} 6$ & $-6.71 \pm 0.07$ & $-7.63 \pm 0.13$ & -6.67 & mas \\
\hline$\Delta \Psi$ (out) Per $18 .^{a} 6$ & $3.76 \pm 0.11$ & $2.91 \pm 0.07$ & 3.65 & mas \\
\hline Per $18^{a} 6$ & $2.64 \pm 0.05$ & $2.86 \pm 0.03$ & 2.86 & mas \\
\hline$\Delta \epsilon$ (out) Per $18{ }^{a} 6$ & $1.37 \pm 0.03$ & $1.49 \pm 0.05$ & 1.55 & mas \\
\hline$\Delta \Psi$ (in) Per $182^{d} \cdot 6$ & $1.63 \pm 0.02$ & $1.64 \pm 0.04$ & 1.69 & mas \\
\hline$\Delta \Psi$ (out) Per $182 .{ }^{d} 6$ & $-1.19 \pm 0.02$ & $-1.42 \pm 0.04$ & -1.40 & mas \\
\hline Per $182{ }^{d} 6$ & $-0.60 \pm 0.01$ & $-0.55 \pm 0.01$ & -0.54 & mas \\
\hline$\Delta \epsilon$ (out) Per $182^{d} 6$ & $-0.24 \pm 0.01$ & $-0.46 \pm 0.01$ & -0.46 & mas \\
\hline$\Delta \Psi$ (in) Per $13^{d} 66$ & $-0.27 \pm 0.02$ & $-0.31 \pm 0.04$ & -0.32 & mas \\
\hline$\Delta \Psi$ (out) Per $13^{d} 66$ & $0.20 \pm 0.02$ & $0.34 \pm 0.04$ & 0.27 & mas \\
\hline Per $13^{d} 66$ & $0.131 \pm 0.007$ & $0.15 \pm 0.01$ & 0.164 & mas \\
\hline$\Delta \epsilon$ (out) Per $13^{d} 66$ & $0.127 \pm 0.007$ & $0.17 \pm 0.01$ & 0.136 & mas \\
\hline
\end{tabular}

\section{Conclusion}

It is shown that an empirical expansion of harmonic variations in Earth rotation can be obtained in one step.

- The advantages of the proposed approach are

- A uniform treatment of harmonic variations in the Earth rotation at diurnal and semidiurnal frequencies;

- No $\Delta \psi, \Delta \epsilon$ time series;

- An ability to estimate correctly short-period nutation amplitudes.

- The disadvantage is high computation cost.

\section{References}

Souchay, J., Feissel, M., Bizouard, C., Capitane, N., Bougeard, M., 1995, Astron. Astrophys. Sup., 299, 277.

McCarthy, D.D. (ed.) IERS Conventions, 1996. 

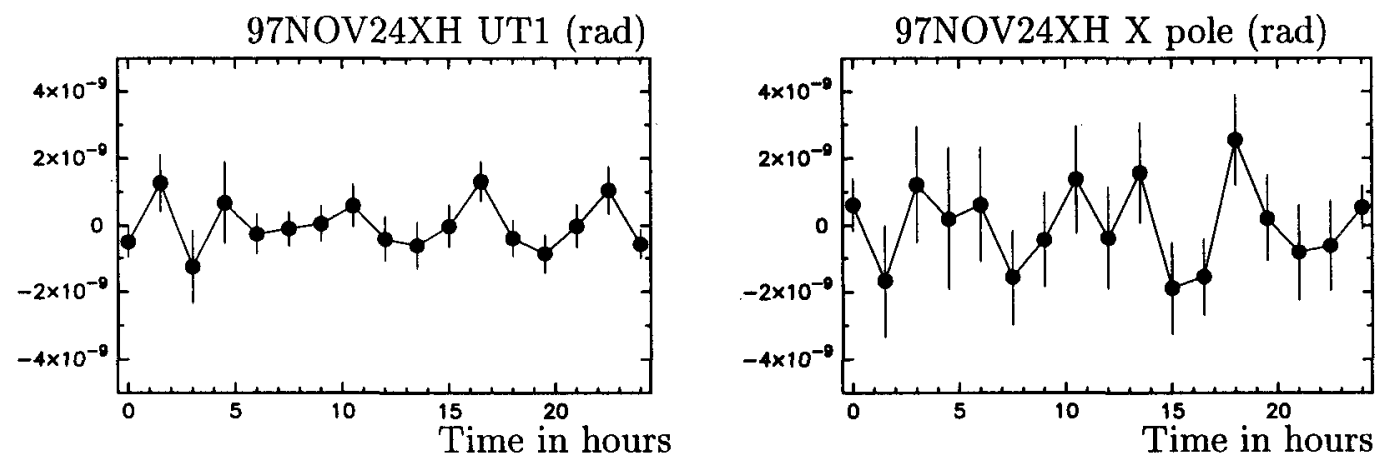

Figure 1. Residual variations in the Earth rotation for a routine VLBI experiment 